\title{
Pulmonary Candidiasis Associated with Severe COVID-19: Case Report
}

Romina Georgina Bórtoli ${ }^{1 *}$, Beatriz Carolina Ascencio Goméz ${ }^{2}$, Carmen Elena Albanez Ayala ${ }^{3}$, Alejandro José Duarte Cuellar ${ }^{4}$, Luis Alberto Camputaro ${ }^{5}$, Laura Estela Miranda Iraheta ${ }^{6}$, Manuel Bello Quesada ${ }^{7}$, Paolo Antoine Vigne Cuellar ${ }^{8}$

${ }^{1}$ Physician, Intensive Care Department. Hospital Nacional El Salvador.San Salvador-El Salvador.

${ }^{2}$ Resident Physician, Intensive Care Department. Hospital Nacional El Salvador. San Salvador-El Salvador.

${ }^{3}$ Infectology Service. Coordinator of the Antimicrobial Optimization Program Team, and Infection Control Coordinator Hospital Nacional El Salvador. San Salvador-El Salvador.

${ }^{4}$ Epidemiologist. Expert Government of El Salvador. Official representative of the Government of El Salvador Global Initiative for Access to Vaccines (GAVI), WHO, PAHO and COVAX.

${ }^{5}$ Physician, Head of Teaching and Research Department. Hospital Nacional El Salvador. San Salvador-El Salvador.

${ }^{6}$ Director. Hospital Nacional El Salvador. San Salvador-El Salvador.

${ }^{7}$ Physician, Head of the Intensive Care Department. Hospital Nacional El Salvador. San Salvador-El Salvador.

${ }^{8}$ Student, fifth year medical degree. Universidad Jose Matias Delgado. San Salvador-El Salvador.

*Corresponding Author: Romina Georgina Bórtoli, Physician, Intensive Care Department. Hospital Nacional El Salvador.San Salvador-El Salvador.

\section{Received Date: August 21, 2021 | Accepted Date: October 09, 2021 | Published Date: October 14, 2021}

Citation: Bórtoli RG, Goméz BCA, Ayala CEA, Cuellar AJD, Camputaro LA, et al. (2021) Pulmonary Candidiasis Associated with Severe COVID-19: Case Report. International Journal of Clinical Case Reports and Reviews. 9(1); DOI:10.31579/2690-4861/166

Copyright: () 2021 Romina Georgina Bórtoli, This is an open-access article distributed under the terms of the Creative Commons Attribution License, which permits unrestricted use, distribution, and reproduction in any medium, provided the original author and source are credited.

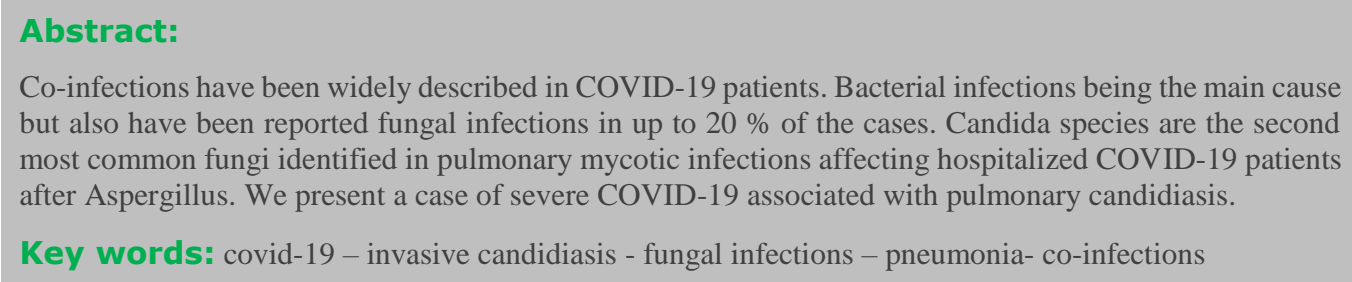

\section{Introduction}

Co-infections have been widely described in COVID-19 patients. Bacterial infections being the main cause but also have been reported fungal infections in up to $20 \%$ of the cases [1-2]. Candida species are the second most common fungi identified in pulmonary mycotic infections affecting hospitalized COVID-19 patients after Aspergillus [3]. We present a case of severe COVID-19 associated with pulmonary candidiasis.

Since the beginning of the COVID-19 pandemic co-infections have been frequently reported, mostly in hospitalized patients with moderate disease (2 - 27\%), but also in patients with severe or critical COVID-19 (14 $58 \%$ ), even more in the deceased $(50 \%)$ [4]. Health-care associated infections are the cause of an increase in morbidity and mortality in patients with or without coronavirus infection.
The new coronavirus has caused an overload of the healthcare systems worldwide, initially all the effort was conducted to this new disease management. We adapted to this new situation by thoroughly research of the infection mechanisms and its complications, including bacterial and fungal co-infections.

Despite of advances in public health and hospital care, infections still emerge in hospitalized COVID-19 patients. Several microorganisms have been identified as co-pathogens, the bacteria are the most frequent isolated, and in less proportion fungal species. To date, the impact of the latter has not been entirely studied.

Patients with COVID-19 in the intensive care unit (ICU) are at high risk of developing healthcare-associated infections due to the requirement for invasive procedures. In these patients the main risk factors for developing invasive candidemia are: prolonged hospital stay, central venous 
catheters, surgical procedures and the use of broad-spectrum antibiotics [5].

\section{Case Report}

A 65-year-old female with previous diagnosis of diabetes mellitus, arterial hypertension and chronic kidney disease presented to a peripheral hospital in San Salvador with a 15-day history of fever, headache, dry cough, and progressive dyspnea. Upon admission SARS COV 2 pneumonia was diagnosed. Treatment was initiated according to the national protocol for moderate COVID-19, which at the time included: zinc, ascorbic acid, vitamin $\mathrm{D}$, dexamethasone and anticoagulation with enoxaparin. Imipenem was also started due to a suspected co-infection. A positive urine culture for E. coli (sensitive to carbapenems, amikacin, ceftriaxone and levofloxacin) was reported, so a 4-days antibiotic treatment was completed.

Four days after hospitalization she was referred to our institution and admitted to the ICU due to severe respiratory failure. The patient required oxygen support with a high-flow nasal cannula to achieved an improvement in oxygen saturation and respiratory mechanics.

\begin{tabular}{|l|c|}
\hline Age & 65 \\
\hline Sex & Female \\
\hline ICU stay (days) & 14 \\
\hline Days on mechanical ventilation & 12 \\
\hline Days under deep sedation in ICU & 10 \\
\hline Quick SOFA & 1 (RR: $24 \mathrm{rpm})$ \\
\hline
\end{tabular}

* Quick SOFA: Quick Sequential Organ Failure Assessment; RR: respiratory rate; rpm: respirations per minute.

Table 1: Description of the patient.

\section{Clinical course}

Laboratory exams on admission presented anemia, leukocytosis with neutrophilia, renal failure, elevated D-dimer and positive non-quantitative troponin (see Table 2). Chest X-ray showed diffuse interstitial-alveolar infiltrates, mainly localized in right lung apex and inferior lobe, without presence of consolidations or evidence of pleural effusion. (Figure 1)

Treatment according to the national protocol for COVID-19 was continued in our institution. Antibiotic therapy with levofloxacin was started for persistent suspicion of sepsis secondary to bacterial coinfection; antiviral treatment with remdesivir was also added.

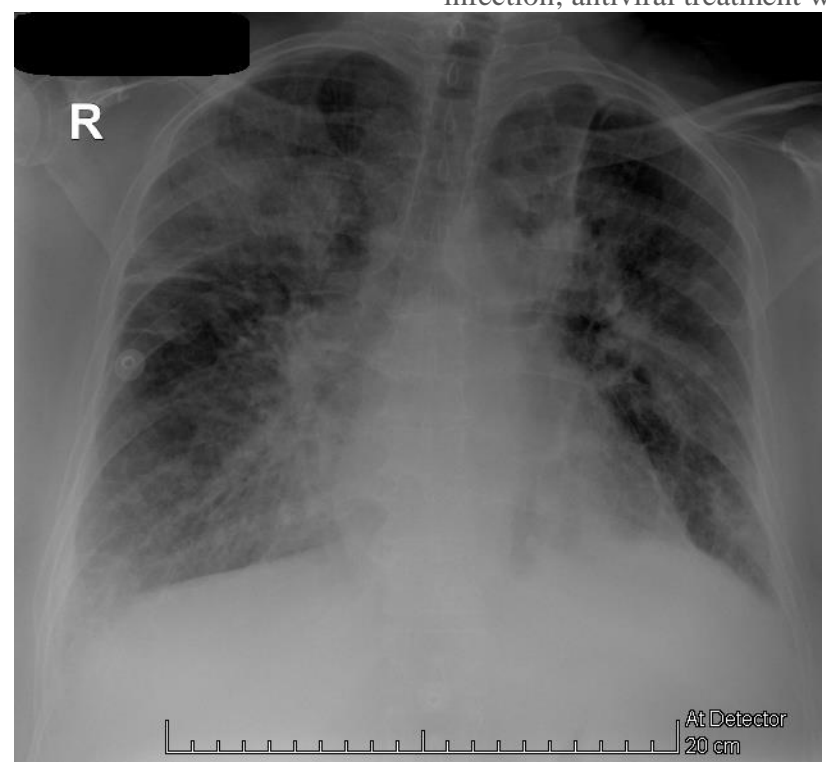

Figure 1: Chest $X$-ray at admission

The third day of hospitalization in our institution the patient had a 7 minutes cardiopulmonary arrest (CA). Non-ST segment elevation acute coronary syndrome was determined as probable CA cause by reason of the multiple cardiovascular risk factors. Post-CA studies showed acute ischemic changes in ECG with positive troponins, for which medical treatment was initiated. In the post-CA care, the patient required invasive mechanical ventilation and sedation analgesia; no need of vasopressors support as the patient present hemodynamic stability. In laboratory exams presented an increased in leucocytes account, suspecting a new nosocomial septic event could be another determinant of the clinical deterioration therefore antibiotic therapy was switch to piperacillin / tazobactam.
The following days, the patient present persistent increase in leukocytosis and required vasopressors as an evidence of organ dysfunction progression despite the use of broad-spectrum antibiotics. Given these clinical findings, an infection resistant to the antibiotics used so far was suspected and treatment with imipenem and vancomycin was started.

Despite broad-spectrum antibiotic treatment, covering possible gramnegative and positive bacteria, radiological deterioration was evidenced as a right apex consolidation, and increase in inflammatory markers in laboratory exams (Figure 2). After receiving a positive result in bronchial aspirate culture for Candida sp, the Infectious Disease Department suspended the antibiotic therapy and started treatment with fluconazole. 


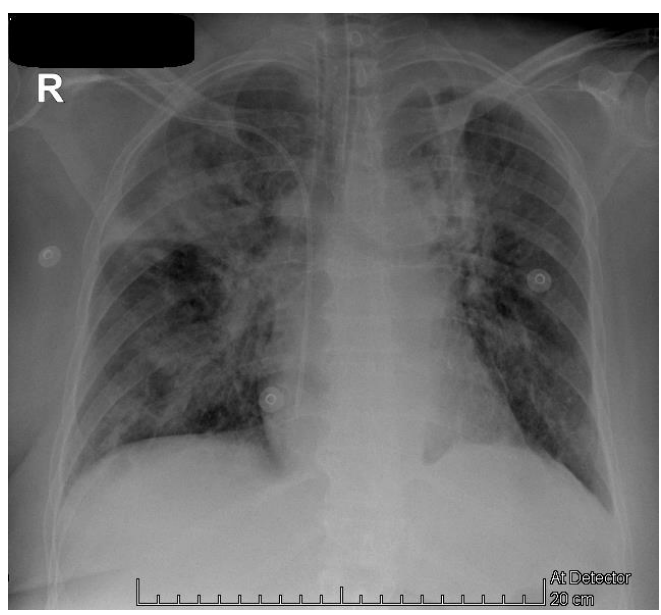

Figure 2: Chest X-ray. Fourth day of admission after an episode of cardiopulmonary arrest requiring mechanical ventilation, it shows right apex pneumonia, in respiratory culture grew Candida Spp.

Forty eight hours after starting fluconazole, a clinical improvement was evidenced, presented decrease of inflammatory markers and a significant reduction of norepinephrine dose. Furthermore, radiological and oxygenation recovery allowed to reduced mechanical ventilation support.(Figure 3).

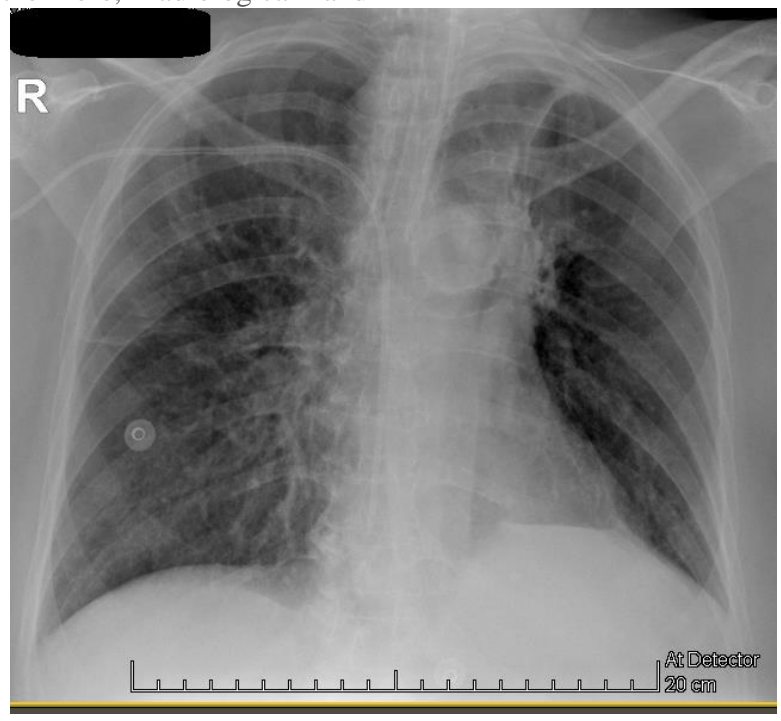

Figure 3: Chest X-ray 48 hours after the start of fluconazole treatment. Show right apex consolidation resolution

As result of the patient clinical condition improvement, progressive sedation de-scalation was performed until suspension, maintaining fentanyl at an analgesic dose. After sedation weaning it was possible to perform a complete neurological examination and a poor neurological response was evidenced (FOUR scale: 5) without sings of focal alterations. In consequence, the mechanical ventilation weaning turned out to be difficult. Unfortunately, the patient died a week after due to complications of post cardiac arrest syndrome.

\begin{tabular}{|l|c|c|c|c|c|}
\hline & $\mathbf{2 / 8 / 2 1}$ & $\mathbf{2 / 1 1 / 2 1}$ & $\mathbf{2 / 1 2 / 2 1}$ & $\mathbf{2 / 1 5 / 2 1}$ & $\mathbf{0 2 / 1 7 / 2 1}$ \\
\hline Hgb (gr/dL) & 7.2 & 8.9 & 10.1 & 8.8 & 9.6 \\
\hline Hct. (\%) & 23.8 & 28.6 & 34.2 & 29.3 & 29.2 \\
\hline Plt. (10e/uL) & 623 & 457 & 434 & 372 & 208 \\
\hline WBC (10e/uL) & 14.67 & 21.69 & 23890 & $\mathbf{3 3 6 7 0}$ & $\mathbf{1 9 , 2 3 0}$ \\
\hline Neutrophils (\%) & $92.2 \%$ & $93.8 \%$ & $96.5 \%$ & $95 \%$ & $90.4 \%$ \\
\hline Leucocytes (\%) & 5.7 & 2.0 & 0.6 & & 2.6 \\
\hline Na (mEq/L) & 136.19 & 144.07 & 145.3 & 148 & \\
\hline
\end{tabular}




\begin{tabular}{|c|c|c|c|c|c|}
\hline $\mathbf{K}(\mathbf{m E q} / \mathbf{L})$ & 5.73 & 4.27 & 4.2 & & \\
\hline $\mathrm{Cl}(\mathrm{mEq} / \mathrm{L})$ & 103.7 & 109.67 & 106.33 & & \\
\hline Creat. $(\mathrm{mg} / \mathrm{dL})$ & 2.21 & 1.77 & 1.95 & 2.78 & 2.6 \\
\hline BUN (g/dL) & 49.29 & 57.78 & 67.3 & 85 & \\
\hline Procalcitonin (ng/ml) & & & 1.17 & 1.2 & \\
\hline IL-6 (pg/mL) & 633.73 & 44.89 & & & \\
\hline CRP (mg/L) & 133.63 & 68.87 & 83.4 & & 35.3 \\
\hline Ferr. (ng/ml) & 53.61 & & & & \\
\hline LDH (UI/L) & 466 & 281 & & & \\
\hline Glucose (mg/dL) & 269.63 & & & & \\
\hline $\mathrm{Ca}(\mathrm{mg} / \mathrm{dL})$ & 8.8 & 8.3 & & & \\
\hline $\operatorname{Mg}(\mathrm{mg} / \mathrm{dL})$ & 1.9 & 1.97 & & 1.56 & \\
\hline TB $(\mathrm{mg} / \mathrm{dL})$ & 0.41 & & & & \\
\hline DB (mg/dL) & 0.14 & & & & \\
\hline IB (mg/dL) & 0.27 & & & & \\
\hline $\operatorname{ALT}(\mathbf{U I} / \mathbf{L})$ & 215 & 107 & & & \\
\hline AST (UI/L) & 178 & 195 & & & \\
\hline D dimer (mg/L FEU) & 4.92 & & & & \\
\hline TP (sec.) & 15.1 & & & & \\
\hline INR & 1.42 & & & & \\
\hline PTT (sec.) & 33.5 & & & & \\
\hline Albumin (g/dL) & & & 2.85 & & 2.99 \\
\hline
\end{tabular}

Hgb: hemoglobin; Hct.: hematocrit; Plt.: platelet count; WBC: white blood cells count; Na: Sodium; K: potassium; Cl: Chloride; Creat.: creatinine; BUN: blood urea nitrogen; IL-6: interleucine-6; CRP: C-reactive protein; Ferr.: Ferritine; LDH: Lactate dehydrogenase; Ca: calcium; Mg: Magnesium; TB: Total Bilirrubin; DB: Direct Bilirrubin; IB: Indirect bilirrubin; ALT: Alanine aminotransferase; AST: Aspartate aminotransferase, PT: prothrombin time; INR: international normalized ratio; PTT: Partial thromboplastin time. *Start of Fluconazole

Table 2: Flow chart laboratory tests.

\begin{tabular}{|l|c|c|c|c|}
\hline Sample date & Result date & Blood culture & Urine culture & Tracheal aspirate culture \\
\hline $02 / 11 / 21$ & $02 / 12 / 21$ & & Negative & \\
\hline $02 / 11 / 21$ & $02 / 15 / 21$ & & & Candida spp. \\
\hline $02 / 11 / 21$ & $02 / 18 / 21$ & Negative & & \\
\hline $02 / 22 / 21$ & $02 / 23 / 21$ & & Negative & \\
\hline
\end{tabular}

Table 3: Culture flowchart

\section{Discussion:}




\section{Invasive Candidiasis in COVID-19}

Microorganism of the Candida genus are part of the resident microbiota of the genitourinary and gastrointestinal tracts. However, when an imbalance in the ecological niche occurs, they can cause infections. Candidiasis is the most common invasive fungal infection associated with medical care, with recent global estimates reporting 750,000 cases of invasive candidiasis (IC) per year and more than 50,000 deaths. [6]

Clinical expression of Candida species infection varies from localized symptoms of the mucous membranes to generalized spread with multisystemic organ failure. [7]. Invasive candidiasis is a rare disease but is associated with a 30-day mortality rate over $35 \%$ in critically ill patients.
Primary Candida pneumonia is an extremely rare disease, described in less than $1 \%$ of cases [8]. Its pathophysiology is not well known, but it is likely associated with hematogenous spread rather than direct contamination due to aspiration. In a monocentric study of patients with mechanical ventilation, only $8 \%$ of the tracheal aspirate samples were positive for Candida, considering that $92 \%$ was colonization thus the colony count did not exceed the diagnosis threshold for mechanical ventilation associated pneumonia [9].

The incidence of candidemia is related to age, with the highest rates observed at the two ends of life. Various risk factors for the development of candidemia have been identified, allowing for the development of scores to determine the possibility of presenting a candida infection. (Table 3) [6].

Critical illness

Long-term ICU stays

Presence of central vascular catheter, total parenteral nutrition

Use of broad-spectrum antibiotics

Presence of central vascular catheter, total parenteral nutrition

Glucocorticoid use

Hemodialysis

Abdominal surgery, with particular risk among patients who have anastomotic leakage or have had repeat

laparotomies

Acute necrotizing pancreatitis

Solid-organ tumors

Hematologic malignant disease

Solid-organ transplantation

Chemotherapy for cancer

Table 3. Risk Factors for Invasive Candidiasis (IC)

The regulation of the host's immune response to the virus is one of the main pillars of COVID-19 treatment, as well as one of the potential risk factors for candida infection in these patients. Some of these treatments include immunosuppressive drugs as glucocorticoids, which have shown to decrease mortality, and also drugs that inhibit IL-6 action. Furthermore, critically ill patients require: prolonged hospitalization, central venous catheters and broad-spectrum antibiotic therapy, which at the beginning of the pandemic was excessive many of the protocols initially included antibiotic coverage at admission], all of these are risk factors for developing invasive infections caused by diverse Candida species [5].

The reported incidence of invasive candidiasis [IC] in COVID ranges from 0.7 to $23 \%$. The pathophysiological mechanisms of COVID-19 are not fully understood, making it difficult to establish the pathogenesis of associated candida infections. The immunocompromise state caused by the coronavirus does not appear to be related to the immunity pathways intervening in candida infections, an example is the lymphopenia found in severe COVID patients, which is not associated to invasive candidiasis. Therefore, the incidence of candida infections is related to the usual risk factors and not to a specific immunocompromise state [5].

Primary Candida pneumonia is considered a rare disease at present, being little information regarding its pathophysiology available, hence the importance of reporting this clinical case. Colonization of the airway by Candida increases with the duration of mechanical ventilation, being $20 \%$ in the first 48 hours. Up to $90 \%$ of COVID patients who require hospitalization receive antibiotic therapy, which can increase the rate of colonization. Damage to the lung epithelium by viral invasion and the inflammatory response could facilitate the passage of candida into the bloodstream. Another possibility is Candida translocation from the gastrointestinal tract in patients who develop sepsis or septic shock [10]. The diagnosis of invasive Candida infections is challenging, specific characteristics of this type of infection such as transient fungemia, low fungal load in cultured tissues, not using specific culture media for fungi routinely or the need of invasive procedures for taking samples, makes it difficult to achieved microbiological isolation.

A positive blood culture is considered the Gold Standard for the diagnosis of candidemia. [11], but is a low sensitivity method, which varies from 21 to $71 \%$ according to the different reports. The high incidence of negative blood cultures resides in the short period of candida in the bloodstream [10]. Currently, alternative methods are available, which include clinical scores, molecular platforms, PCR, panels, which together achieve up to $80 \%$ sensitivity. However, it can be increased if the negative predictive value of low procalcitonin is use to rule out a bacterial infection, as presented in our patient laboratory tests [12].

A prompt diagnosis and treatment are the key to reduce mortality. In patients with COVID, mortality was higher in those who did not receive antifungals.

Treatment: The medical management of Invasive Candidiasis follow the same methodology for patients with or without COVID. In general, antifungal therapy should be started immediately after positive blood cultures and an active search for possible metastatic foci is necessary, such as endocarditis, retinal and deep liver or splenic abscesses [12]. Likewise, if there is a high probability of deep candida infection and blood cultures are negative, the diagnosis should not be ruled out.

Early antifungal therapy and source control are the most important interventions for the treatment of IC, with better results as earlier therapy is started [11]. Effective antifungal agents against Candida include echinocandins, azoles and amphotericin B. [13] Nevertheless sensitivity varies depending on the species of Candida, being resistance to azoles more frequent in non-albicans species. 
In general, monotherapy is used in antifungal treatment, since no benefits have been found when using a combination of antifungals [14]. The optimal time therapy should last for candidemia is not fully established. For patients with candidemia in the absence of metastatic complications, a minimum treatment of two-weeks is suggested after blood cultures become negative. [15]

Empirical antifungal therapy: In the absence of positive blood cultures or evidence of deep tissue invasive candidiasis, the approach to empirical antifungal therapy depends on individual clinical circumstances [16].

\section{Conclusion:}

Candida pneumonia is a rare complication in hospitalized patients. The importance of this pathology and others invasive candidiasis in outcomes y mortality in COVID-19 patients is matter of further study.

\section{Conflicts of Interest}

The authors declare that they have no conflicts of interest.

The publication of the case report was approved by the Committee of Ethics in Investigation Hospital Nacional El Salvador.

\section{Funding}

This case report did not have financial support.

\section{References}

1. White P, Dhillon R, Cordey A, Hughes H, Faggian F et al. (2020). A national strategy to diagnose COVID-19 associated invasive fungal disease in the ICU. Clin Infect Dis.

2. Lansbury L, Lim B, Baskaran V, Lim WS. (2020). Coinfections in people with COVID-19: a systematic review and meta-analysis. J Infect. 81(2):266-275.

3. Cox M, Loman N, Bogaert D, O'Grady J. (2020). Coinfections: potentially lethal and unexplored in COVID-19. Lancet Microbe.

4. Al-Hatmi A, Mohsin J, Al-Huraizi A, Khamis F. (2021). COVID-19 associated invasive candidiasis. J Infect. 82(2):4546.
5. Arastehfar A, Carvalho A, Nguyen M et al. (2020). COVID-19Associated Candidiasis (CAC): An Underestimated Complication in the Absence of Immunological Predispositions? J Fungi (Basel). 6(4):211.

6. Kullberg B, Arendrup M. (2015). Invasive Candidiasis. N Engl J Med. 373(15):1445-1456.

7. Carol A Kauffman. (2021). Candida infections of the abdomen and thorax. En: UpToDate, Post TW (Ed), UpToDate, Waltham, MA.

8. Kontoyiannis D, Reddy B, Torres H et al. (2002). Pulmonary candidiasis in patients with cancer: an autopsy study. Clin Infect Dis. 34(3):400-403.

9. Wood G, Mueller E, Croce M et al. (2006). Candida sp. isolated from bronchoalveolar lavage: clinical significance in critically ill trauma patients. Intensive Care Med. 32(4):599-603.

10. Wood GC, Mueller EW, Croce MA et al. (2006). Candida sp. isolated from bronchoalveolar lavage: clinical significance in critically ill trauma patients. Intensive Care Med. 32(4):599603.

11. Carol A Kauffman, MD. (2021). Clinical manifestations and diagnosis of candidemia and invasive candidiasis in adults. En: UpToDate, Post TW (Ed), UpToDate, Waltham, MA.

12. Pappas P, Lionakis M, Arendrup $M$ et al. (2018). Invasive candidiasis. Nat Rev Dis Primers. 4:18026.

13. Kollef M, Micek S, Hampton N et al. (2012). Septic shock attributed to Candida infection: importance of empiric therapy and source control. Clin Infect Dis. 54(12):1739-1746.

14. Rex JH, Pappas PG, Karchmer AW, et al. (2003). A randomized and blinded multicenter trial of high-dose fluconazole plus placebo versus fluconazole plus amphotericin B as therapy for candidemia and its consequences in non neutropenic subjects. Clin Infect Dis. 36(10):1221-1228.

15. Pappas PG, Kauffman CA, Andes DR et al. (2016). Clinical Practice Guideline for the Management of Candidiasis: 2016 Update by the Infectious Diseases Society of America. Clin Infect Dis. 62(4):1-50.

16. Gonzalez-Lara MF, Ostrosky-Zeichner L. (2020). Invasive Candidiasis. Semin Respir Crit Care Med. 41(1):3-12.

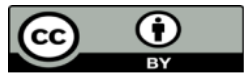

This work is licensed under Creative Commons Attribution 4.0 License

To Submit Your Article Click Here: Submit Manuscript

DOI: $10.31579 / 2690-4861 / 166$
Ready to submit your research? Choose Auctores and benefit from:

$>$ fast, convenient online submission

$>$ rigorous peer review by experienced research in your field

$>$ rapid publication on acceptance

$>$ authors retain copyrights

$>$ unique DOI for all articles

$>$ immediate, unrestricted online access

At Auctores, research is always in progress.

Learn more auctoresonline.org/journals/international-journal-of-clinicalcase-reports-and-reviews 